\title{
A Posteriori-Derived Dietary Patterns and Retinal Vessel Caliber in an Elderly Population
}

\author{
Claire T. McEvoy, ${ }^{1}$ Christopher R. Cardwell, ${ }^{1}$ Usha Chakravarthy, ${ }^{2}$ Ruth E. Hogg, ${ }^{2}$ \\ Michelle C. McKinley, ${ }^{1}$ Ian S. Young, ${ }^{1}$ Astrid E. Fletcher, ${ }^{3}$ and Jayne V. Woodside ${ }^{1}$
}

Purpose. To examine the association between a posterioriderived dietary patterns (DP) and retinal vessel caliber in an elderly population.

Methods. This was a cross-sectional study of 288 elderly adults ( $>65$ years) who participated in the European Eye study (EUREYE) Northern Irish cohort. DP were extracted using principal component analysis from completed food frequency questionnaires. Semi-automated computer grading was used to determine the mean retinal vessel diameters (central retinal arteriole equivalent [CRAE] and central retinal venule equivalent [CRVE]) from digitized visual field one images using a standard measurement protocol.

REsults. Three major DP were identified in this population, which accounted for $21 \%$ of the total variance: a "healthy" pattern with high factor loadings for oily fish, fruits and vegetables, and olive oil; an "unhealthy" pattern with high factor loadings for red and processed meat, refined grains, eggs, butter, sugar and sweets; and a "snack and beverage" pattern with high factor loading for pizza, nuts, and coffee. Multivariable linear regression analysis indicated no significant association between major identified DP and mean CRAE or CRVE in all models.

Conclusions. This is the first study to investigate associations between a posteriori-derived DP and retinal vessel caliber. There was no evidence of a relationship between extracted DP and retinal vessel measurements in this population. However, it is possible that potentially important relationships exist between single nutrients or foods and vessel diameters that cannot be identified using a DP approach. Further studies to examine the role of dietary factors in the microcirculation are required. (Invest Ophthalmol Vis Sci. 2013;54:1337-1344) DOI:10.1167/iovs.12-11065

$\mathrm{D}$ ietary pattern (DP) analysis captures the complexity of overall dietary intake and accounts for cumulative and interactive effects of single nutrients and foods as consumed by individuals, ${ }^{1}$ which can be related to disease outcome(s). DP

From the ${ }^{1}$ Centre for Public Health and the ${ }^{2}$ Centre for Vision and Vascular Science, Queen's University Belfast, Belfast, United Kingdom; and the ${ }^{3}$ Faculty of Epidemiology and Population Health, London School of Hygiene \& Tropical Medicine, London, United Kingdom.

Submitted for publication October 2, 2012; revised December 21, 2012; accepted January 4, 2013.

Disclosure: C.T. McEvoy, None; C.R. Cardwell, None; U. Chakravarthy, None; R.E. Hogg, None; M.C. McKinley, None; I.S. Young, None; A.E. Fletcher, None; J.V. Woodside, None

Corresponding author: Jayne V. Woodside, Centre for Public Health, School of Medicine, Dentistry \& Biomedical Sciences, Queen's University Belfast, Institute of Clinical Science B (First Floor), Grosvenor Road, Belfast, BT12 6BJ, United Kingdom; j.woodside@qub.ac.uk can be examined using two main approaches; a posteriori patterns are empirically derived from the data in hand and are independent of their relevance to health, whereas a priori pattern analysis is a score-based approach that determines the quality of dietary intake based on current hypotheses regarding the role of dietary factors in disease prevention. ${ }^{2}$ A posteriori analyses employ exploratory methods, namely principal component analysis (PCA)/factor analysis or cluster analysis (CA), which aggregate variables into factors representing patterns of food consumption relevant to the study population. Factor scores or clusters can then be examined in relation to disease outcomes or biomarkers of disease. Previous prospective studies involving elderly populations have mainly focused on examining PCA-derived DP and mortality risk. ${ }^{3-10}$ All studies, with the exception of one, ${ }^{5}$ have reported lower risk of all cause or cardiovascular mortality in the highest category of a posteriori-derived dietary pattern, broadly perceived as healthy, relative to the lowest category.

In older adults, microvascular disease has been implicated as a pathogenic factor in cardiovascular disease (CVD) ${ }^{11}$ and is potentially reversible. ${ }^{12}$ The microvasculature of the retina can be viewed noninvasively and provides a unique opportunity to objectively assess measures of vascular health such as vessel diameter. Changes in retinal vessels have been previously shown to be associated with diseases that affect the systemic vasculature. ${ }^{13}$ In elderly men and women, wider retinal venules have been independently associated with incident CVD, while narrower retinal arterioles have been independently associated with incident coronary heart disease (CHD) and hypertension. ${ }^{14}$

To date, no study has examined a posteriori-derived DP in relation to microvascular health in elderly populations. The aim of this study was to examine the association between major PCA-derived DP and retinal vessel caliber in an elderly population.

\section{Methods}

We exploited the availability of a bank of retinal fundus images with corresponding lifestyle, dietary, and blood antioxidant data that had been acquired from participants in the European Eye study (EUREYE). EUREYE is a multicenter, population-based, cross-sectional study involving seven European countries (Norway, Estonia, United Kingdom, France, Italy, Greece, and Spain) and the study methodology and its outcomes have been published in detail. ${ }^{15-17}$ EUREYE was conducted according to the guidelines laid down in the Declaration of Helsinki and ethical approval was granted in each of the study centers.

The EUREYE data repository contains information on demographic, lifestyle and anthropometric variables, dietary intake, and serum analysis of vitamins and carotenoids. The subset of fundus images captured in the UK site (which recruited participants in Northern Ireland (NI) with examinations in this cohort completed in 2002) was used in this study. 


\section{Study Participants}

Fundus images were available in 684 participants from the NI site. We scrutinized the dataset and selected the image from one eye only of 313 participants where field 1 (centered on the optic disc) was clearly visible. Seventeen of these were deemed to be ungradable due to unsatisfactory image quality, resulting in 294 suitable images for analysis. Dietary intake was not available for an additional six participants within the selected cohort; therefore, 288 participants were included in the analysis.

\section{Assessment of Dietary Intake}

Dietary intake was assessed in EUREYE using the European Prospective Investigation into Cancer and Nutrition (EPIC) 196-item, semiquantitative food frequency questionnaire (FFQ) ${ }^{18}$ In the NI center, the questionnaire was modified to reflect only foods relevant to the UK diet $^{19}$ and has been previously validated. ${ }^{20}$ The modified FFQ consisted of 133 items, incorporating a nine-category frequency of consumption scale and standard portion size estimates. Participants were asked to record the frequency of consumption over the past year of each listed food item on a daily, weekly, or monthly basis. Portion sizes of consumed foods were converted to daily gram weight using Crawley's food portion sizes..$^{21}$ Estimates of energy and nutrient intakes from food and beverages were calculated using a computerized dietary analysis program based on the UK food composition tables (WISP; Tinuviel Software, Warrington, UK). The individual FFQ food items were grouped according to shared nutrient or food profiles into 39 food groups as shown in Table 1. Some food items were considered individually as their food/nutrient characteristics were unique (e.g., butter, tofu, tea, coffee, chips, pizza, highenergy drinks). The identified food groups were used to derive dietary pattern by PCA analysis.

\section{Assessment of Anthropometric, Demographic, and Lifestyle Variables}

Participants completed an interview-administered questionnaire in order to ascertain medical history including history of diabetes, hypertension, angina, stroke, MI, or other CVD and lifestyle information regarding smoking status, alcohol intake, and medication use. Weight was recorded to the $0.1 \mathrm{~kg}$. Demi-span was measured in a horizontal plane, from the sternal notch to the web of the ring finger from the left outstretched arm, using a flexible tape measure and recorded in $\mathrm{cm}$. Body mass index (BMI) was calculated as weight $(\mathrm{kg}) /$ height $(\mathrm{m})^{2}$ in which height was calculated from demi-span using equations derived from a substudy in the Spanish EUREYE population. ${ }^{22}$ Systolic and diastolic blood pressure $(\mathrm{mm} \mathrm{Hg})$ were measured in the seated position after 5 minutes of rest using a blood pressure monitor (Omron HEM 705 CP; Omron Healthcare UK Ltd., Milton Keynes, England). Two consecutive readings were obtained with a 5minute interval between measurements. The average of the two readings was considered the participant's blood pressure. Hypertension was classified as having a systolic/diastolic blood pressure greater than 140/90 mm Hg. A nonfasting blood sample was taken in order to quantify plasma levels of micronutrients.

\section{Assessment of Retinal Vessel Caliber}

For the present study, field 1 images in the right eye were selected for vessel measurement. If retinal vessel caliber could not be measured in the right eye, the left was chosen for measurement. Fundus images (field 1 centered on the optic disk and field 2 macula) had been captured using a digital camera (Topcon TRC 50 EX; Topcon, Tokyo, Japan) with a field size of $35^{\circ}$ and flash setting of $50 \mathrm{~mW}$. A single trained technician captured all fundus images within the NI EUREYE center according to a standardized protocol. ${ }^{23}$ Images were submitted to a single reading center by participating technicians in each site for accreditation.
Retinal vessel diameters were measured using a semiautomated software program (IVAN; University of Wisconsin-Madison, Madison, WI). The program was used to demarcate the average optic disc diameter for each image and define grid areas 0.0 to 0.5 (zone A) and 0.5 to 1.0 (zone B) disc diameters from the optic disc margin as shown in the Figure. All retinal vessels $>25 \mu \mathrm{m}$ caliber coursing through zone $B$ were tracked by the software using microdensitometry. The software program used an algorithm to compute the mean diameter of the specified vessel along its length. A trained observer identified the vascular segments in zone B as either arterioles or venules. Only the six largest arteriole and six largest venule diameter measurements were combined in an iterative process of pairing up the largest with the smallest vessels using the formulae ${ }^{24}$ :

$$
\begin{aligned}
& \text { Arterioles: } W=0.88^{*}\left(w_{1}^{2}+w_{2}^{2}\right)^{0.5} \\
& \text { Venules: } W=0.95^{*}\left(w_{1}^{2}+w_{2}^{2}\right)^{0.5}
\end{aligned}
$$

where $\mathrm{w}_{1}$ and $\mathrm{w}_{2}$ are the diameters of the largest and the smallest of the six largest identified vessels, respectively.

All vessel diameters were paired in the same way, using five iterations for six vessel measurements, until a single figure was obtained. This figure was the central retinal arteriole equivalent (CRAE)/central retinal venule equivalent (CRVE), which are summary variables representing the retinal arteriole and venule caliber. The arteriovenous ratio (AVR) was also calculated as the ratio of the arteriole caliber in relation to venule caliber (CRAE/CRVE). An AVR of 0.80 suggested that the arteriole caliber is approximately $80 \%$ as large as the venule caliber

A Posteriori-Dietary Pattern Analysis. PCA/factor analysis method was used to determine factor loadings for the 39 food groups after orthogonal (varimax) transformation to produce uncorrelated factors. The number of factors to retain was determined by examination of the scree plot and based upon the number of points above the breakpoint. Variables with factor loadings $\geq 0.4$ on a component were considered informative in describing the DP and used to interpret the factors. Factor scores were computed for each participant by summing intakes of food groups weighted by their factor loadings and each participant received a factor score for each identified dietary pattern. Participants were then categorized by quartile of dietary pattern score.

\section{Laboratory Analysis}

Serum concentrations of carotenoids (lutein, zeaxanthin, $\beta$-cryptoxanthin, $\alpha$-carotene, $\beta$-carotene, and lycopene) were determined by reverse phase high-performance liquid chromatography (HPLC) with diode array detection according to Craft. ${ }^{25}$ Plasma ascorbate (vitamin C) was measured by automated fluorimetric assay on a centrifugal analyzer (Cobas FARA; Roche Diagnostics, West Sussex, UK) with a fluorescent attachment according to Vuilleumier and Keck. ${ }^{26}$ Intraassay coefficients of variation (CV) were $<7 \%$ for each analyte, and interassay CVs $<10 \%$.

\section{Statistical Analysis}

Differences in general characteristics across quartile categories of dietary pattern score were assessed using descriptive statistical tests. One-way ANOVA with Tukey's post-hoc comparison was used for continuous variables and $\chi^{2}$ test was used for categorical variables. Energy adjusted means for dietary variables were calculated across quartiles of dietary pattern. Skewed variables were logarithmically transformed prior to analysis. Pearson's correlation coefficient was used to determine associations between micronutrient intake and micronutrient status. A general linear model was used to adjust mean retinal vessel summary variables for covariates (e.g., age, sex, smoking, systolic blood pressure). Multivariable linear regression analysis was used to determine the relationship between identified DP and retinal 
TABLE 1. Food Grouping Used in the DP Analysis

\begin{tabular}{|c|c|}
\hline Food Groups & Food Items Included \\
\hline Red meat & Beef, pork, lamb, beef burgers, lasagna \\
\hline Organ meat & Liver/liver pate/liver sausage \\
\hline Poultry & Chicken and other poultry \\
\hline Processed meat & Bacon, ham, savory pies, corned beef/spam/luncheon meats/sausages. \\
\hline White fish and shellfish & Cod/haddock/plaice/sole/halibut, crab/prawn/mussels, fish fried in batter \\
\hline Oily fish & Mackerel/kippers/tuna/salmon/sardines/herring \\
\hline Refined grains & White bread and rolls, white rice, white or green pasta \\
\hline Whole grains & Porridge, brown bread/whole-meal bread and rolls, whole-meal pasta, brown rice \\
\hline Breakfast cereals & Cornflakes/muesli, etc. \\
\hline Potatoes & Boiled/mashed/instant/jacket/roast/salad \\
\hline Chips & Chips \\
\hline Crisps & Crisps and other packet snacks \\
\hline Pizza & Pizza \\
\hline Low fat dairy & Low fat yogurt/fromage frais, cottage cheese/low fat soft cheese, semi- skimmed milk, skimmed milk \\
\hline High fat dairy & Full fat yogurt, whole milk, cheese, single cream, double/clotted cream, condensed milk \\
\hline Eggs & Eggs, quiche \\
\hline $\begin{array}{l}\text { Dressings/sauces/ } \\
\text { condiments }\end{array}$ & Mayonnaise, salad cream, pickles/chutney, marmite/Bovril, white/cheese sauce/gravy \\
\hline Butter & Butter \\
\hline Processed fat/margarine & Block margarine, soft margarine, low fat spread \\
\hline Desserts & Biscuits, cakes, milk pudding, ice cream \\
\hline Chocolate & Chocolate, chocolate snack bars, hot chocolate/cocoa, horlicks/Ovaltine \\
\hline Sugar and sweets & Sugar, sweets/toffees/mints, jam/marmalade/honey \\
\hline Nuts & Peanut butter, peanuts and other nuts \\
\hline Soups & Vegetable/meat soups \\
\hline Tea & Tea \\
\hline Coffee & Instant/ground, decaffeinated coffee, coffee whitener \\
\hline Wine & White, red \\
\hline Beer & Beer/lager/cider \\
\hline Other alcoholic drinks & Port/sherry/vermouth/liqueurs, spirits \\
\hline High energy drinks & Fizzy soft drinks \\
\hline Low energy drinks & Diet fizzy drinks, squash \\
\hline Fruit juice & Pure fruit juice, orange juice, tomato juice \\
\hline Fruit & $\begin{array}{l}\text { Grapefruit, apples, pears, oranges, melons, Satsuma, banana, grapes (white and red), peaches/plums/apricots, } \\
\text { tinned fruit, dried fruit, strawberries/raspberries }\end{array}$ \\
\hline & Kiwi \\
\hline Vegetables & Mushrooms, bean sprouts, coleslaw, cucumber, parsnip/turnip/swedes, beetroot, sweet peppers (red/orange) \\
\hline L/Z-rich vegetables* & Carrots, sweet peppers (green), sweet corn, celery, avocado, lettuce, marrow/courgettes \\
\hline \multirow{2}{*}{$\begin{array}{l}\text { Cruciferous/green leafy } \\
\text { vegetables }\end{array}$} & Broccoli, spring greens, kale, cabbage, cauliflower, brussels sprouts, spinach \\
\hline & Watercress \\
\hline Legumes & Peas, French beans, broad beans, baked beans, dried lentils \\
\hline Allium family & Onions, leeks, garlic \\
\hline Tomato & Tomatoes, tomato products \\
\hline Soya meat & Tofu \\
\hline
\end{tabular}

${ }^{*} \mathrm{~L} / \mathrm{Z}$ rich, lutein-/zeaxanthin-rich vegetables.

vessel caliber. All regression models were adjusted for age and sex. Subsequent analyses adjusted for smoking (current/past/never); systolic blood pressure ( $\mathrm{mm} \mathrm{Hg}$ ); diabetes (absent/present); history of CVD (absent/present); BMI $\left(\mathrm{kg} / \mathrm{m}^{2}\right)$; alcohol intake (never/occasionally/weekly); the alternative retinal vessel caliber measurement for CRAE/CRVE summary variables $(\mu \mathrm{m})$; and energy intake (kcals/day). The first quartile of dietary pattern was used as the reference category. All analyses were performed using statistical analysis software (SPSS, version 17; SPSS, Chicago, IL).

\section{Results}

\section{Subject Characteristics}

EUREYE participants excluded from this study $(n=396)$ were on average 1.2 years older $(P=0.01)$, with greater prevalence of existing CVD (23\% vs. $17 \% ; P=0.007)$ and lower measured systolic $(P=0.01)$ and diastolic $(P<0.0001)$ blood pressure when compared with participants with valid retinal vessel caliber measurement included in this analysis $(n=288$; data not shown).

Characteristics of the EUREYE participants, with valid assessment of retinal vessel caliber, are shown in Table 2 . The population included 152 (53\%) males and 136 (47\%) females with a mean age of 73.5 years, ranging from 66 to 93 years. According to BMI classification, approximately, $4 \%$ of the study participants were underweight $\left(\mathrm{BMI}<19 \mathrm{~kg} / \mathrm{m}^{2}\right) ; 33 \%$ were normal weight (BMI $\geq 19$ and $<25 \mathrm{~kg} / \mathrm{m}^{2}$ ); $33 \%$ were overweight (BMI $\geq 25$ and $<30 \mathrm{~kg} / \mathrm{m}^{2}$ ); and $30 \%$ were clinically obese (BMI $\geq 30 \mathrm{~kg} / \mathrm{m}^{2}$ ). Approximately $14 \%$ were current smokers, $43 \%$ were former smokers, and $43 \%$ had never smoked. Almost $75 \%$ of the population consumed 


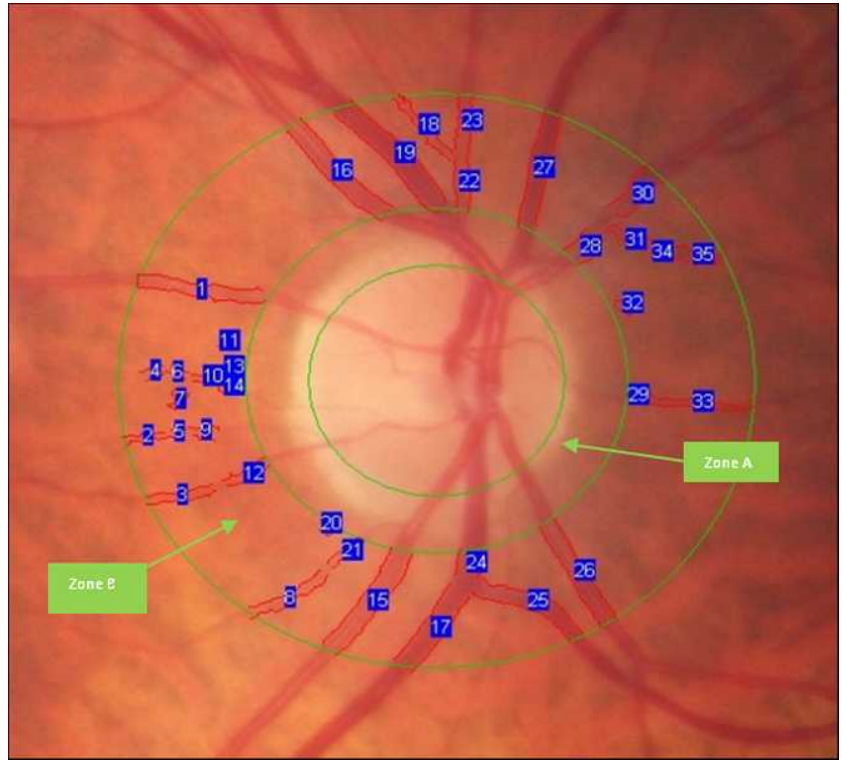

Figure. Fundus photograph capturing a field one image of the right eye (centered on the optic disc) indicating zone A (0.0-0.5 optic disc diameter) and zone B (0.5-1.0 optic disc diameter).

alcohol either regularly or occasionally. In addition, $13 \%$ of the population had been previously diagnosed with diabetes and $17 \%$ had at least one CVD event. Almost half (49\%) of the population also reported a previous diagnosis of hypertension, while $31 \%$ had evidence of uncontrolled blood pressure, defined as mean measured systolic/diastolic blood pressure $>140 />90 \mathrm{~mm} \mathrm{Hg}$. Mean CRAE and CRVE was 157.1 and 225.4 $\mu \mathrm{m}$, respectively. Mean AVR was 0.71, suggesting that arterioles were on average $70 \%$ as large as the venule caliber in this elderly population.

\section{PCA-Derived DP}

Three major DP (eigenvalues $<2$ ) were identified using PCA/ factor analysis and we labeled them "healthy," "unhealthy," and "snack and beverage." The factor loading matrices for these major DPs are shown in Table 3. The healthy pattern had high factor loadings for oily fish, all fruits and vegetables, and olive oil. The unhealthy pattern had high factor loadings for red and processed meat, refined grains, eggs, butter, sugar and sweets. The snack and beverage pattern had high factor loadings for pizza, nuts, and coffee. Overall, the three major identified DPs explained approximately $21 \%$ of the total variance. There were no differences in age, sex, prevalence of obesity, diabetes, CVD, or hypertension across quartiles of healthy or unhealthy dietary pattern score (data not shown). In the complete sample, total energy adjusted carotene intake was significantly correlated with total carotenoid micronutrient status $(r=0.135 ; P=0.03$ [using Pearson's correlation coefficient]). Furthermore, there was a significant linear increase in total carotenoid status between the lowest and the highest fourths of the healthy pattern $(P$ for trend $=0.008)$ and a linear decrease in total carotenoid status between the lowest and highest fourths of the unhealthy pattern ( $P$ for trend $=0.005$ ). There was a significant linear decrease in plasma vitamin $C$ concentrations between the lowest and the highest fourth in the unhealthy pattern $(P$ for trend $=0.03$ ). However, this observation is limited by the small numbers of plasma vitamin C samples analyzed $(n=53$ in sample; data not shown).
TABLE 2. General Characteristics of the EUREYE Study Participants with Valid Assessment of Retinal Vessel Caliber $(n=288)$

\begin{tabular}{|c|c|c|}
\hline Characteristic & Mean (SD) & Range \\
\hline Age, y & $73.5(5.3)$ & 66 to 93 \\
\hline Sex, male $n(\%)^{*}$ & $152(52.8)$ & \\
\hline Weight, kg & $75.0(14.3)$ & 38 to 117 \\
\hline BMI, $\mathrm{kg} / \mathrm{m}^{2}$ & $27.3(5.6)$ & 12.3 to 44.6 \\
\hline \multicolumn{3}{|l|}{ Smoking status* } \\
\hline Current, $n$ (\%) & $42(14.5)$ & \\
\hline Former, $n(\%)$ & $114(39.5)$ & \\
\hline Never, $n(\%)$ & $113(39.2)$ & \\
\hline \multicolumn{3}{|l|}{ Alcohol intake* } \\
\hline At least weekly, $n$ (\%) & $93(32.3)$ & \\
\hline Occasionally, $n$ (\%) & $121(42.0)$ & \\
\hline Never, $n(\%)$ & $74(25.7)$ & \\
\hline $\mathrm{CVD}, n(\%)^{*}$ & $49(17)$ & \\
\hline Diabetes, $n(\%)^{*} \dagger$ & $37(12.9)$ & \\
\hline HTN, $n(\%)^{*}$ & $140(48.6)$ & \\
\hline $\mathrm{SBP} / \mathrm{DBP}>140 / 90(\mathrm{~mm} \mathrm{Hg}), \dagger n(\%)$ & $83(31.0)$ & \\
\hline CRAE, $\mu \mathrm{m}$ & $157.1(17.0)$ & 111.9 to 211.8 \\
\hline CRVE, $\mu \mathrm{m}$ & $225.4(24.4)$ & 159.3 to 295.3 \\
\hline AVR & $0.71(0.11)$ & 0.44 to 1.12 \\
\hline
\end{tabular}

CVD, previous cardiovascular disease (heart disease or stroke); HTN, reported hypertension; SBP, systolic blood pressure; DBP, diastolic blood pressure.

* Categorical variables summarized as $n$ (\%).

† Available for 286 and 268 participants, respectively, and therefore percent values summarized as valid percent.

\section{Major Determinants of Retinal Vessel Caliber}

In this study population, univariate analysis using a general linear model indicated different systemic determinants of measured CRAE and CRVE. For CRAE, a sex difference in arteriole diameter was observed: on average, males had arterioles $4.3 \mu \mathrm{m}$ narrower than females $(P=0.04)$. Similarly, each $1 \mathrm{~mm} \mathrm{Hg}$ increase in systolic blood pressure was associated with narrower arterioles by an average $0.1 \mu \mathrm{m}(P$ $=0.04)$. In contrast, there were no observed associations between sex and SBP and measured CRVE. However, increased energy intake was associated with wider venules; each $1 \mathrm{kcal}$ increase in energy was associated with $0.01 \mu \mathrm{m}$ wider venules $(P=0.04)$. Intake of monounsaturated fatty acid (MUFA) was also positively related to CRVE: each $1 \mathrm{~g}$ increase in MUFA was associated with an average $2.5 \mu \mathrm{m}$ widening in venule diameter $(P=0.04)$.

There were no further associations observed between retinal vessel summary variables and other covariates, including age (years); alternate retinal vessel diameter variable $(\mu \mathrm{m})$; frequency of alcohol intake (never/occasionally/weekly); smoking status (current/past/never); CVD (absent/present); diabetes (absent/present); diastolic blood pressure ( $\mathrm{mm} \mathrm{Hg}$ ); or BMI $\left(\mathrm{kg} / \mathrm{m}^{2}\right)$.

We found no significant difference in retinal vessel summary variables across quartiles of the three major DP scores as shown in Table 4. A significant difference in arteriole diameter was observed between Q1 and Q4 in the adjusted healthy pattern: those in the highest fourth having on average $6.5 \mu \mathrm{m}$ (95\% confidence interval [CI]: $0.3,12.7)$ narrower arterioles than those in the lowest fourth $(P=0.04)$, after adjustment for all recorded potential confounders (see Table 4). However, no significant linear trend was observed across quartiles of the healthy DP. Multivariable linear regression analysis indicated no significant association between major identified DP and measured retinal vessel diameter in all adjusted models as shown in Table 4. 
TABLE 3. Factor Loading Matrix* for the Three Major DPs Identified from FFQ

\begin{tabular}{lccc}
\hline \multicolumn{1}{c}{ Food Groups } & \multicolumn{3}{c}{ Food Patterns Identified } \\
\cline { 2 - 4 } \multicolumn{1}{c}{ Red meat } & Healthy & Unhealthy & Snack and Beverage \\
Processed meat & - & 0.456 & - \\
Oily fish & - & 0.500 & - \\
Refined grains & 0.495 & - & - \\
Pizza & - & 0.562 & - \\
Eggs & - & - & -479 \\
Butter & - & 0.413 & - \\
Sugar and sweets & - & 0.537 & - \\
Nuts & - & 0.540 & - \\
Tea & - & - & -0.456 \\
Coffee & - & - & 0.664 \\
Vegetables & - & - & - \\
L/Z-rich vegetables* & 0.678 & - & - \\
Cruciferous/green leafy vegetables & 0.736 & - & - \\
Legumes & 0.485 & - & - \\
Allium & 0.567 & - & - \\
Tomato & 0.598 & - & - \\
Olive oil & 0.530 & - & - \\
Fruit & 0.494 & 0.447 & - \\
\hline
\end{tabular}

${ }^{*}$ Values $<0.4$ were excluded for simplicity.

\section{Discussion}

To our knowledge, this is the first study to examine whole DP in relation to retinal vessel caliber measurement. Three major DPs were identified in this population using PCA/factor analysis: a healthy dietary pattern, an unhealthy dietary pattern, and a snack and beverage dietary pattern. We found no evidence of an association between these major identified PCA-derived DP and retinal vessel diameters in an elderly population.

The present study is novel in that it uses PCA/factor analysis to derive DP. The nature of a posteriori DP analysis means that the patterns are derived from the data in-hand without a priori assumptions. Therefore, derived DP reflects the combination of cultural, ethnic, and socioeconomic influences on eating behavior specific to the study population. The healthy and unhealthy patterns we found are similar to those previously reported in other populations. ${ }^{27,28}$ We also validated our findings by showing that serum carotenoids increased across quartiles of healthy pattern scores and decreased with increasing unhealthy dietary pattern score.

Other studies examining PCA-derived DP in relation to established measures of subclinical vascular health-such as carotid intima-media thickness (CIMT), coronary artery calcium, and pulse wave velocity - have also found only weak or no associations in middle-aged to older populations after adjustment for potential confounders. ${ }^{29-31}$ In contrast, significant associations between single foods or nutrients and subclinical atherosclerosis in middle-aged to older populations have been shown. Intake of saturated fat has been positively related to CIMT $^{32}$ while intakes of fruit, dietary fiber, and soy foods have been independently inversely related to CIMT in different population groups. ${ }^{33-35}$ Single foods and nutrients have also been associated with retinal vessel caliber. Increased intakes of dietary fiber and fish have been independently and significantly associated with wider arteriole caliber and narrower venule caliber. ${ }^{36-38}$ While current evidence is limited to crosssectional observation, it is possible that specific nutrients and/or foods may have more influence on the microvasculature than overall dietary intake. If this is the case, the DP approach, although a potentially useful way of capturing the complexity of overall diet, may dilute any effect of a specific nutrient/ food, ${ }^{39}$ and therefore the true association between dietary factors and retinal vessel measurement may be obscured.

We found no relationship between self-reported CVD and retinal vessel caliber in this study. In general, associations between retinal vessel abnormalities and incident CVD in older populations are weaker than that reported in middle-aged adults. ${ }^{40}$ An age-related decrease in predictive value of retinal vessel abnormalities for CVD risk has been suggested, which may relate to differences in disease comorbidity, adverse CVD risk factor profiles, and differences in pathologic mechanisms associated with the aging process, ${ }^{40}$ and may have contributed to the lack of association observed in the present study. Increasing blood pressure is strongly and consistently associated with retinal arteriolar narrowing. Likewise, we too found that systolic blood pressure was a significant determinant of narrower arterioles in this elderly population: each $1 \mathrm{~mm} \mathrm{Hg}$ increase in systolic blood pressure was associated with a 0.1 $\mu \mathrm{m}$ narrowing in arteriole diameter, which is consistent with published findings in this area. ${ }^{41}$ We also found a significant sex effect on retinal arteriole caliber, with females having wider arterioles than men, in accordance with other cohort studies. ${ }^{41,42}$ We found no significant systemic determinants of retinal venule caliber in our population. However, both energy intake and MUFA intake were positively related to wider venules: a novel finding that requires validation in further studies.

Limitations of the present study include the inherent drawbacks of the DP approach. Although PCA has been shown to have good reproducibility and validity when applied to FFQ data, ${ }^{39}$ the method is based on a number of subjective arbitrary decisions regarding the selection of food grouping, the method of rotation, the number of retained factors, and the amount and naming of dietary pattern categories, which can contribute to overall measurement error. It is noteworthy that the major DPs identified in this study explained $21 \%$ of the total variance, suggesting that unidentified eating patterns may be associated with vascular health in this population-although this figure is similar to other studies using PCA to define dietary patterns. $^{7,8,27}$ 


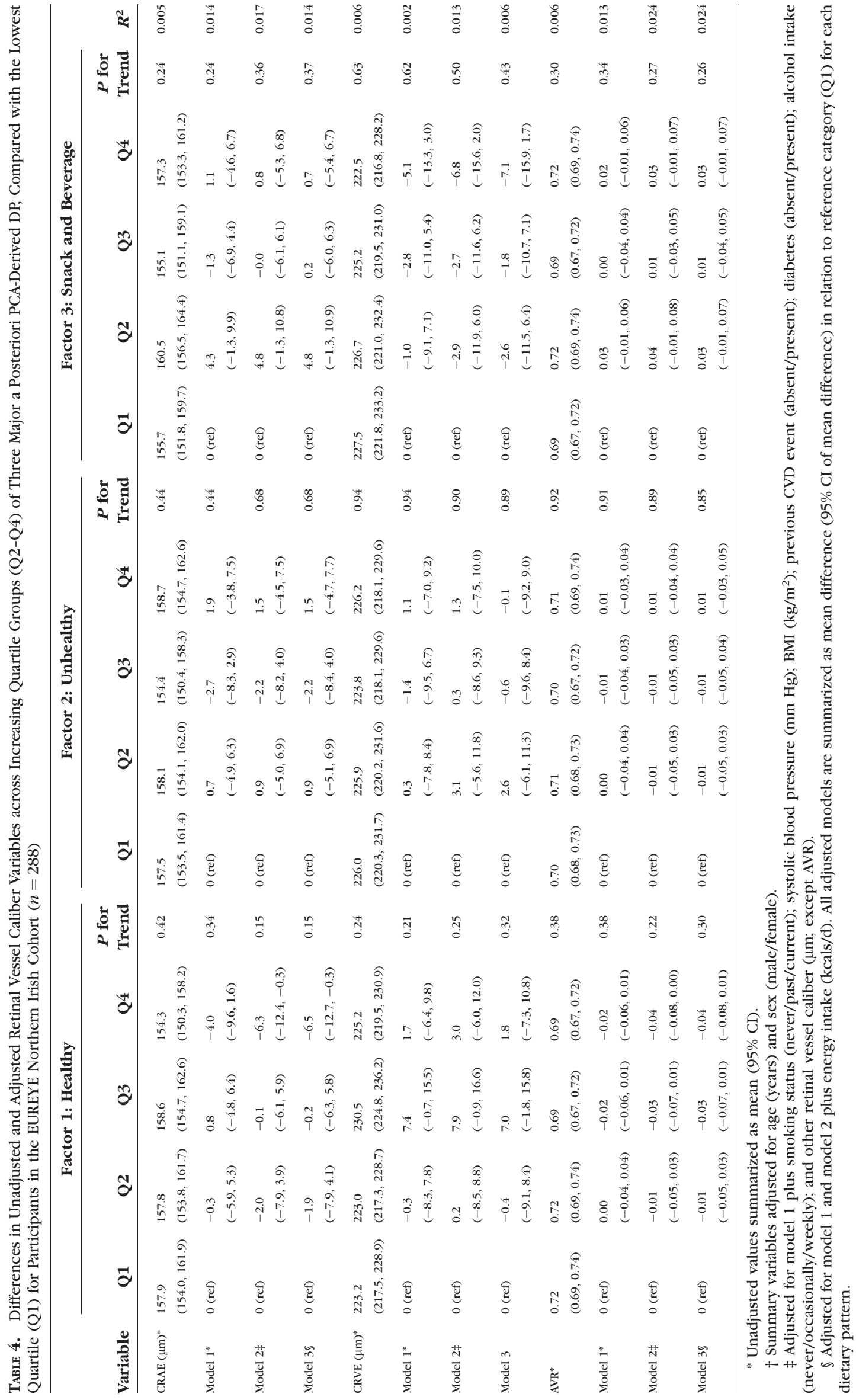


We were also unable to establish a temporal relationship between DP and retinal vessel caliber owing to the crosssectional study design, the single measure of dietary intake, and the inclusion of participants with and without preexisting CVD and CVD risk factors. Dietary misclassification is possible as participants may have changed their lifetime eating behavior as a result of symptomatic disease. Paradoxically, we found that those in the highest quartile of healthy pattern had significantly narrower arterioles compared with the lowest quartile. Furthermore, our major identified DP did not relate to lifestyle variables such as BMI, smoking status, or alcohol intake, which contrasts with other prospective studies examining PCAderived DP and disease outcomes. ${ }^{7,8,27,28}$ Overall, these findings may indicate that identified DPs in our population are reflective of more recent eating behavior and may not fully account for lifetime dietary exposure. Furthermore, the sample size of the current study is smaller compared with observational studies examining retinal vessel caliber and CVD outcome, which may have resulted in reduced statistical power to detect differences in vessel diameters. In addition, the possibility of measurement error exists as there are several potential sources of variation in the quantification of retinal vessel caliber, despite adherence to a strict measurement protocol similar to that used in large prospective studies. The reliability of this method to assess retinal vessels has been previously reported as high, with intergrader weighted kappa of 0.85 and 0.90 and intragrader weighted kappa between 0.80 and 0.93 for arteriolar and venular caliber measurements, respectively. ${ }^{43}$ Unfortunately, we were unable to determine intergrader variability due to time and cost limitations of the current study.

In summary, we found no evidence of an association between PCA-derived DP and retinal vessel caliber in an elderly population. There are no previous studies investigating DP and retinal vessel caliber; however, our findings largely agree with current limited evidence examining a posteriori-derived DP and established surrogate markers of vascular disease. Further longitudinal prospective and intervention studies are warranted to elucidate the role of DP in relation to vascular disease, particularly in older adults and women with known increased prevalence of the disease.

\section{Acknowledgments}

We thank the EUREYE Steering Committee for their input: Astrid Fletcher (PI), Christina Augood, Usha Chakravarthy, Paulus de Jong, Mati Rahu, Johan Seland, Gisele Soubrane, Laura Tomazzoli, Fotis Topouzis, Jesus Vioque, and Ian Young.

\section{References}

1. Schulze MB, Hoffmann K. Methodological approaches to study dietary patterns in relation to risk of coronary heart disease and stroke. Br J Nutr. 2006;95:860-869.

2. Kant AK. Dietary patterns: biomarkers and chronic disease risk. Appl Physiol Nutr Metab. 2010;35:199-206.

3. Kumagai S, Shibata H, Watanabe $S$, et al. Effect of food intake pattern on all-cause mortality in the community elderly: a 7-year longitudinal study. J Nutr Health Aging. 1999;3:29-33.

4. Diehr P, Beresford SA. The relation of dietary patterns to future survival, health, and cardiovascular events in older adults. J Clin Epidemiol. 2003;56:1224-1235.

5. Hoffmann K, Boeing H, Boffetta P, et al. Comparison of two statistical approaches to predict all-cause mortality by dietary patterns in German elderly subjects. Br J Nutr. 2005;93:709716.
6. Waijers PM, Ocke MC, van Rossum CT, et al. Dietary patterns and survival in older Dutch women. Am J Clin Nutr. 2006;83: 1170-1176.

7. Bamia C, Trichopoulos D, Ferrari P, et al. Dietary patterns and survival of older Europeans: the EPIC-Elderly Study (European Prospective Investigation into Cancer and Nutrition). Pub Health Nutr. 2007;10:590-598.

8. Masala G, Ceroti M, Pala V, et al. A dietary pattern rich in olive oil and raw vegetables is associated with lower mortality in Italian elderly subjects. Br J Nutr. 2007;98:406-415.

9. Hamer M, McNaughton SA, Bates CJ, et al. Dietary patterns, assessed from a weighed food record, and survival among elderly participants from the United Kingdom. Eur J Clin Nutr. 2010;64:853-861.

10. Anderson AL, Harris TB, Tylavsky FA, et al. Dietary patterns and survival of older adults. J Am Diet Assoc. 2011;111:84-91.

11. Chaves PH, Kuller LH, O'Leary DH, et al. Subclinical cardiovascular disease in older adults: insights from the Cardiovascular Health Study. Am J Geriatr Cardiol. 2004;13: 137-151.

12. Leung DY, Leung M. Non-invasive/invasive imaging: significance and assessment of coronary microvascular dysfunction. Heart. 2011;97:587-595.

13. Liew G, Wang JJ. Retinal vascular signs: a window to the heart? Rev Esp Cardiol. 2011;64:515-521.

14. Wong TY, Kamineni A, Klein R, et al. Quantitative retinal venular caliber and risk of cardiovascular disease in older persons: the cardiovascular health study. Arch Intern Med. 2006; 166:2388-2394.

15. Augood CA, Fletcher AE, Bentham GC, et al. Methods for a population-based study of the prevalence of and risk factors for age-related maculopathy and macular degeneration in elderly European populations: the EUREYE study. Ophthalmic Epidemiol. 2004;11:117-129.

16. Augood CA, Chakravarthy U, Young I, et al. Oily fish consumption, dietary docosahexaenoic acid and eicosapentaenoic acid intakes, and associations with neovascular agerelated macular degeneration. Am J Clin Nutr. 2008;88:398406.

17. Fletcher AE, Bentham GC, Agnew M, et al. Sunlight exposure, antioxidants, and age-related macular degeneration. Arch Ophthalmol. 2008;126:1396-1403.

18. Willett WC, Sampson L, Stampfer MJ, et al. Reproducibility and validity of a semiquantitative food frequency questionnaire. Am J Epidemiol. 1985;122:51-65.

19. Fletcher AE, Breeze E, Shetty PS. Antioxidant vitamins and mortality in older persons: findings from the nutrition add-on study to the Medical Research Council Trial of Assessment and Management of Older People in the Community. Am J Clin Nutr. 2003;78:999-1010.

20. Bingham SA, Welch AA, McTaggart A, et al. Nutritional methods in the European Prospective Investigation of Cancer in Norfolk. Pub Health Nutr. 2001;4:847-858.

21. Nelson M, Atkinson M, Meyer JAA. Photographic Atlas of Food Portion Sizes. London: MAFF Publications; 1997:1-102.

22. van Leeuwen R, Chakravarthy U, Vingerling JR, et al. Grading of age-related maculopathy for epidemiological studies: is digital imaging as good as 35-mm film? Ophthalmology. 2003; 110:1540-1544.

23. Weinbrenner T, Vioque J, Barber X, et al. Estimation of height and body mass index from demi-span in elderly individuals. Gerontology. 2006;52:275-281.

24. Knudtson MD, Lee KE, Hubbard LD, et al. Revised formulas for summarizing retinal vessel diameters. Curr Eye Res. 2003;27: 143-149. 
25. Craft NE. Carotenoid reversed-phase high-performance liquid chromatography methods: reference compendium. Methods Enzymol. 1992;213:185-205.

26. Vuilleumier JP, Keck E. Fluorometric assay of vitamin C in biological materials using a centrifugal analyser with fluorescence attachment. J Micronut Analysis. 1989;5:25-34.

27. Hu FB, Rimm EB, Stampfer MJ, et al. Prospective study of major dietary patterns and risk of coronary heart disease in men. Am J Clin Nutr. 2000;72:912-921.

28. Fung TT, Willett WC, Stampfer MJ, et al. Dietary patterns and the risk of coronary heart disease in women. Arch Intern Med. 2001;161:1857-1862.

29. Kesse-Guyot E, Vergnaud AC, Fezeu L, et al. Associations between dietary patterns and arterial stiffness, carotid artery intima-media thickness and atherosclerosis. Eur J Cardiovas Prev Rehabil. 2010;17:718-724.

30. Millen BE, Quatromoni PA, Nam BH, et al. Dietary patterns, smoking, and subclinical heart disease in women: opportunities for primary prevention from the Framingham Nutrition Studies. J Am Diet Assoc. 2004;104:208-214.

31. Nettleton JA, Steffen LM, Schulze MB, et al. Associations between markers of subclinical atherosclerosis and dietary patterns derived by principal components analysis and reduced rank regression in the Multi-Ethnic Study of Atherosclerosis (MESA). Am J Clin Nutr. 2007;85:1615-1625.

32. Merchant AT, Kelemen LE, de Koning L, et al. Interrelation of saturated fat, trans fat, alcohol intake, and subclinical atherosclerosis. Am J Clin Nutr. 2008;87:168-174.

33. Ellingsen I, Hjerkinn EM, Seljeflot I, et al. Consumption of fruit and berries is inversely associated with carotid atherosclerosis in elderly men. Br J Nutr. 2008;99:674-681.
34. Mellen PB, Liese AD, Tooze JA, et al. Whole-grain intake and carotid atherosclerosis in a multiethnic cohort: The Insulin Resistance Atherosclerosis Study. Am J Clin Nutr. 2007;85: 1495-1502.

35. Buil-Cosiales P, Irimia P, Ros E, et al. Dietary fibre intake is inversely associated with carotid intima-media thickness: a cross-sectional assessment in the PREDIMED study. Eur J Clin Nutr. 2009;63:1213-1219.

36. Kan H, Stevens J, Heiss G, et al. Dietary fiber intake and retinal vascular caliber in the Atherosclerosis Risk in Communities Study. Am J Clin Nutr. 2007;86:1626-1632.

37. Kaushik S, Wang JJ, Flood V, et al. Frequency of fish consumption, retinal microvascular signs and vascular mortality. Microcirculation. 2008;15:27-36.

38. Kaushik S, Wang JJ, Wong TY, et al. Glycemic index, retinal vascular caliber, and stroke mortality. Stroke. 2009;40:206212.

39. Hu FB, Willett WC. Optimal diets for prevention of coronary heart disease. JAMA. 2002;288:2569-2578.

40. Longstreth W Jr, Larsen EK, Klein R, et al. Associations between findings on cranial magnetic resonance imaging and retinal photography in the elderly: the Cardiovascular Health Study. Am J Epidemiol. 2007;165:78-84.

41. Wong TY, Klein R, Klein BE, et al. Retinal vessel diameters and their associations with age and blood pressure. Invest Ophthalmol Vis Sci. 2003;44:4644-4650.

42. Leung H, Wang JJ, Rochtchina E, et al. Relationships between age, blood pressure, and retinal vessel diameters in an older population. Invest Ophthalmol Vis Sci. 2003;44:2900-2904.

43. Liew G, Wang JJ, Mitchell P, et al. Retinal vascular imaging: a new tool in microvascular disease research. Circul Cardiovas Imaging. 2008;1:156-161. 